\title{
Bioökonomie
}

\section{Metabolic Engineering von Cyanobakterien für nachhaltiges Bioplastik}

MORITZ KOCH, KARL FORCHHAMMER

INTERFAKULTÄRES INSTITUT FÜR MIKROBIOLOGIE UND INFEKTIONSMEDIZIN, UNIVERSITÄT TÜBINGEN

\section{Global crises like climate change require the transformation of our industry towards more sustainability. Applying metabolic engineering to cyanobacteria promises to use these phototrophic bacteria for the biosynthesis of carbon-neutral products. As one example, we present how recent advances in basic research have now led to the ability to produce high quantities of bioplastics in cyanobacteria.}

DOI: $10.1007 / \mathrm{s} 12268-021-1541-4$

(C) Die Autoren 2021

Cyanobakterien sind als einzige Bakterien in der Lage, oxygene Photosynthese zu betreiben. Daher können diese Organismen nur mittels $\mathrm{CO}_{2}$ und Sonnenlicht wachsen, ohne organische Kohlenstoffquellen zu verwenden. Darüber hinaus können Cyanobakterien aber noch mehr: Viele Stämme produzieren PHB (Polyhydroxybutyrat). Dahinter verbirgt sich ein Kohlenstoffpolymer, das sich im Cyanobakterien-Modellorganismus Synechocystis sp. PCC 6803 unter unausgeglichen Wachstumsbedingungen, wie etwa Stickstoffmangel, anhäuft [1]. Auch wenn die physiologische Bedeutung von PHB für Cyanobakterien nach wie vor Rätsel aufgibt, ist die mögliche Anwendung für uns Menschen lange bekannt: PHB eignet sich als Rohstoff für Bioplastik. Neben guten Materialeigenschaften, die häufig mit Polypropylen verglichen werden, bie- tet es darüber hinaus eine gute biologische Abbaubarkeit. Dank der $\mathrm{CO}_{2}$-neutralen Produktion in Cyanobakterien kann somit also ein nachhaltiges Produkt auch nachhaltig hergestellt werden [2]. Bislang sind die intrazellulär akkumulierten Mengen an PHB jedoch sehr gering (etwa 10 \% PHB pro Zelltrockengewicht), was eine industrielle Produktion bislang noch unrentabel macht. Deswegen hat sich unsere Arbeitsgruppe zum Ziel gesetzt, den PHB-Metabolismus in Synechocystis besser zu verstehen, um anschließend mittels metabolic engineering die Produktivität zu steigern.

\section{Grundlagen des PHB-Metabolismus in Cyanobakterien}

$\mathrm{Zu}$ Beginn unserer Forschung galt es, die Kultivierungsbedingungen näher zu erfor-

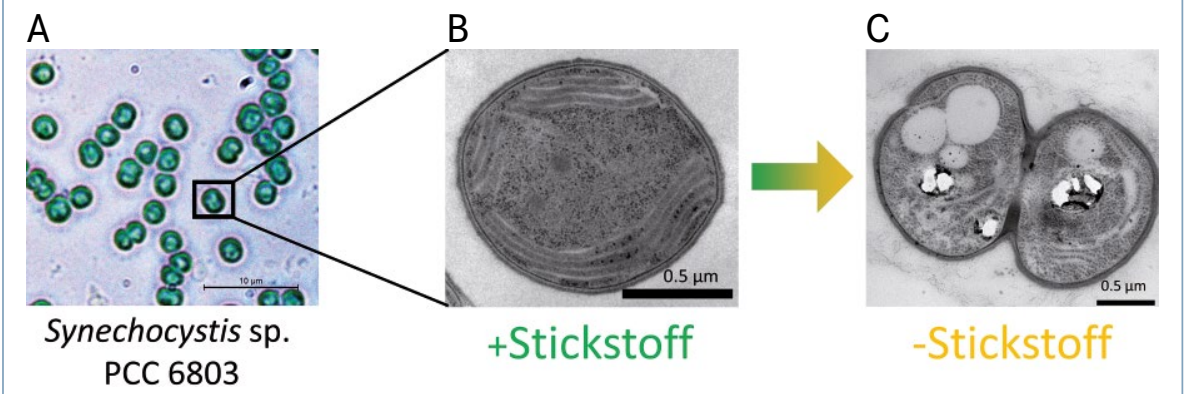

$\Delta$ Abb. 1: Schematische Darstellung des PHB-Biosynthese-Ablaufs. Synechocystis-Kulturen mit ausreichend Stickstoff im Medium im Licht- (A) bzw. Elektronenmikroskop (B). C, Ist kein Stickstoff im Medium vorhanden, initiiert dies die PHB-Biosynthese (helle Granula). schen, unter denen PHB produziert wird. Dabei stellte sich heraus, dass in einer Synechocystis-Kultur die einzelnen Zellen interessanterweise eine große Heterogenität in ihrer Menge an produziertem PHB aufweisen [3]. Es zeigte sich, dass innerhalb einer Population genetisch identischer Bakterien manche Zellen mehr, andere weniger PHB herstellen - ein Befund, der relevant für die gleichmäßige und stabile Produktion ist. Als nächstes galt es, den Aufbau der PHB-Granula und die damit assoziierten Proteine näher $\mathrm{zu}$ verstehen. Im Gegensatz zu etablierten PHB-Produktionsstämmen, wie Cupriavidus necator, von dem über ein Dutzend relevante Proteine bekannt sind, wurden diese in Synechocystis bislang wenig erforscht. Wir konnten mit PhaP und Slr0058 erstmals Proteine identifizieren, die maßgeblich die Anzahl und Größe der PHB-Granula regulieren $[4,5]$. Auch die Frage nach dem Stoffwechselweg des Kohlenstoffs für die PHBSynthese konnten wir aufklären. Studien mit diversen Mutanten zeigten, dass das PHB aus dem Katabolismus von Glykogen stammt [6]. Somit war nun auch die Herkunft des Kohlenstoffs geklärt und ermöglichte die gezielte Veränderung des Stoffwechsels zur Erhöhung der PHB-Synthese.

\section{Mittels metabolic engineering zu maximaler PHB-Akkumulation}

Ein entscheidender Durchbruch gelang in unserem Labor in einem Projekt, das ursprünglich gar nicht mit der PHB-Forschung im Zusammenhang stand. Bei der Untersuchung von Interaktionspartnern des Signalproteins $\mathrm{P}_{\text {II }}$ entdeckten wir mit PirC kürzlich einen zentralen Regulator für die Verteilung des fixierten Kohlenstoffs. PirC kontrolliert die Aktivität des Enzyms Phosphoglycerat-Mutase, das 3-Phosphoglycerat (der erste stabile Metabolit der RuBisCoabhängigen $\mathrm{CO}_{2}$-Fixierung) in 2-Phosphoglycerat umwandelt und dadurch neu fixierten Kohlenstoff aus dem Calvinzyklus entnimmt [7]. In einer Mutante, in der PirC deletiert ist, findet ein verstärkter Glykogen-Abbau und ein erhöhter Stofffluss in Richtung Acetyl- 
Abb. 2: Schematische Darstellung der PHB-Biosynthese in Synechocystis. Unter Stickstoffmangel wird Kohlenstoff in Glykogen gespeichert. Im Laufe des Prozesses wird Glykogen durch den EMP-Weg zu AcetylCoA-Einheiten verstoffwechselt, wobei das Protein PirC ein entscheidender Regulator ist. Acetyl-CoA wird dann in mehreren Schritten und mithilfe von SIr0058 und PhaP zu PHB-Granula verarbeitet.
CoA, dem Baustein für die PHB-Synthese, statt. Durch die Kombination einer PirCMutante mit der Überexpression der PHBBiosynthese-Gene phaAB in eine PirC-basierte Mutante konnten wir so einen Stamm erschaffen (PPT1), der die höchste jemals gemessene PHB-Akkumulation in einem phototrophen Organismus aufweist [8]. Somit steigerten wir die intrazelluläre Menge an PHB von etwa zehn Prozent auf über 80 Prozent pro Zelltrockengewicht. Solche Mengen waren bislang lediglich heterotrophen Organismen wie der Burkholderien-Art Cupriavidus necator vorbehalten. Die akkumulierte Menge an PHB war in Synechocystis-Mutanten schließlich so groß, dass vereinzelt die Zellen aufplatzten. So hat die langjährige Erforschung des Synechocystis-Stoffwechsels zur massiven Steigerung der produzierten PHB-Mengen geführt.

Für die Zukunft gilt es nun, die generierten Stämme weiter zu optimieren und in Pilot- anlagen hochzuskalieren, um Markfähigkeit zu erlangen. Bis dahin ist es wichtig, die gesetzlichen und regulatorischen Rahmenbedingungen zu schaffen, damit nachhaltige,

$\mathrm{CO}_{2}$-neutrale Produkte auch wettbewerbsfähig sind.

\section{Danksagung}

Ein besonderer Dank gilt der Studienstiftung des Deutschen Volkes für das Promotionsstipendium von Moritz Koch und der DFG für kontinuierliche Förderung (speziell dem GRK 1708). Darüber hinaus danken wir allen beteiligten Studierenden, Mitarbeiter/inne/n und Kollaborationspartner/inne/n.

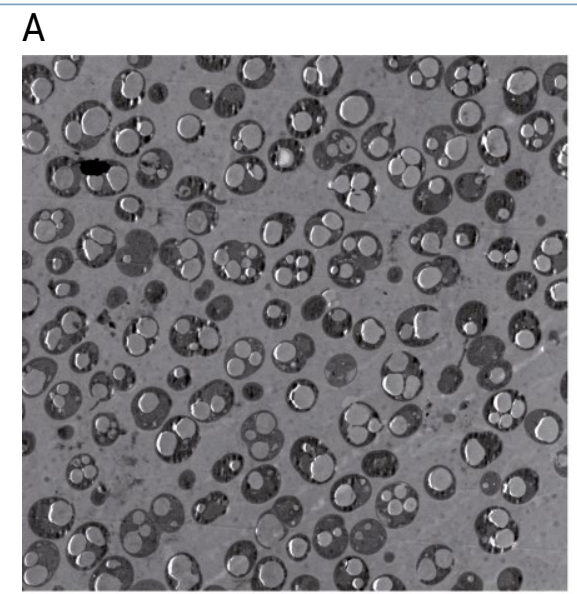

C

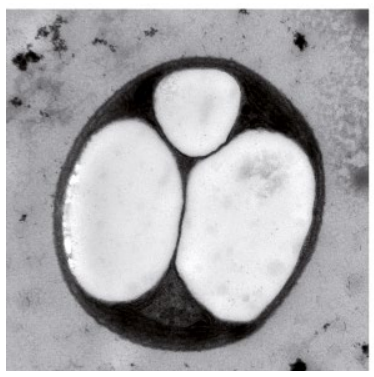

D

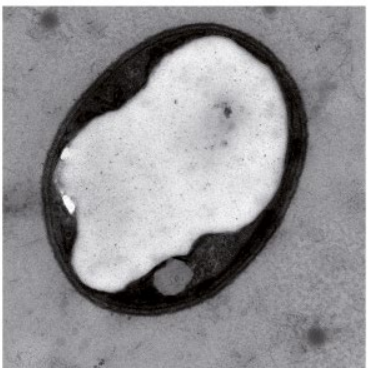

B

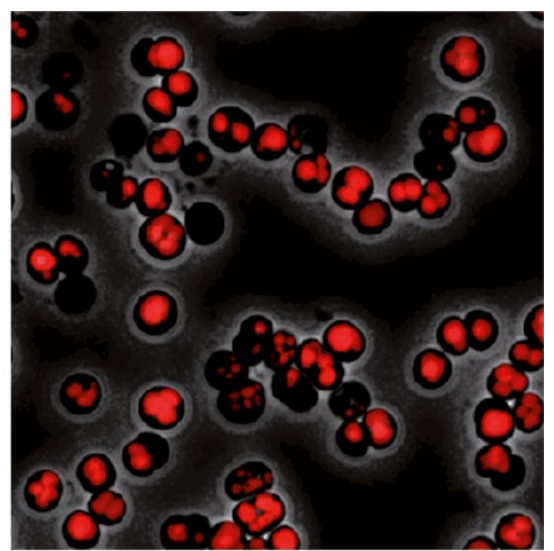

E

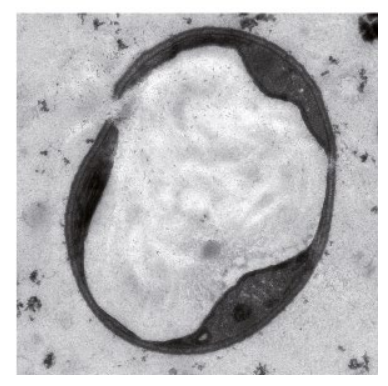

$\triangle$ Abb. 3: PHB-Produktion in der Mutante PPT1. A, Im Elektronenmikroskop zeigen viele Synechocystis-Zellen große Granula. B, Anfärbung mittels Nile-Rot-Farbstoff bestätigt die Granula als PHB. Manche der Zellen enthalten mehrere Granula (C), andere nur ein einziges Granulum (D). E, In manchen Zellen zeigen die TEM-Aufnahmen ein Aufplatzen der Zellhülle. Modifiziert aus [8].

\section{Literatur}

[1] Klotz A, Georg J, Bučinská L et al. (2016) Awakening of a dormant cyanobacterium from nitrogen chlorosis reveals a genetically determined program. Curr Biol 26: 2862-2872

[2] Koller M, Marsalek L (2015) Cyanobacterial polyhydroxyalkanoate production: status quo and quo vadis? Curr Biotechnol 4: 464-480

[3] Koch M, Berendzen K, Forchhammer K (2020) Revealing the physiological role of PHB for cyanobacteria. Life 10: 47

[4] Hauf W, Watzer B, Roos N et al. (2015) Photoautotrophic polyhydroxybutyrate granule formation is regulated by cyanobacterial phasin PhaP in Synechocystis sp. strain PCC 6803. Appl Environ Microbiol 81: 4411-4422

[5] Koch M, Orthwein T, Alforth J T et al. (2020) The Slr0058 Protein From Synechocystis sp. PCC 6803 Is a Novel

Regulatory Protein Involved in PHB Granule Formation. Fron Microbiol 11: 809

[6] Koch M, Doello S, Gutekunst K et al. (2020) PHB is produced from glycogen turn-over during nitrogen starvation in Synechocystis sp. PCC 6803. Int J Mol Sci 20: 1942

[7] Orthwein T, Scholl J, Spät P et al. (2021) The novel PIIinteractor PirC identifies phosphoglycerate mutase as key control point of carbon storage metabolism in cyanobacteria. Proc Natl Acad Sci U S A 118: e2019988118

[8] Koch M, Bruckmoser J, Scholl J et al. (2020) Maximizing PHB content in Synechocystis sp. PCC 6803: a new metabolic engineering strategy based on the regulator PirC. Microb Cell Fact 19: 231

Funding note: Open Access funding enabled and organized by Projekt DEAL. Open Access: Dieser Artikel wird unter der Creative Commons Namensnennun 4.0 International Lizenz veröffentlicht, welche die Nutzung, Vervielfältigung, Bearbeitung, Verbreitung und Wiedergabe in jeglichem Medium und Form ordnungsgemäß nennen, einen Link zur Creative Commons Lizenz beifügen und angeben, ob Anderungen vorgenommen wurden. Die in diesem Artikel enthaltenen Bilder und sonstiges Drittmaterial unterliegen ebenfalls der genannten Creative Commons Lizenz, sofern sich aus der Abbildungslegen nichts anderes ergibt. Sofern das betreffende Material nicht unter der genannten Creative Commons Lizenz stent und die betreffende Handlung nich Weiterverwendungen des Materials die Einwilligung des jeweiligen Rechteinhabers einzuholen. Weitere Details zur Lizenz entnehmen Sie bitte der Lizenzinformation auf http://creativecommons.org/licenses/by/4.0/deed de.
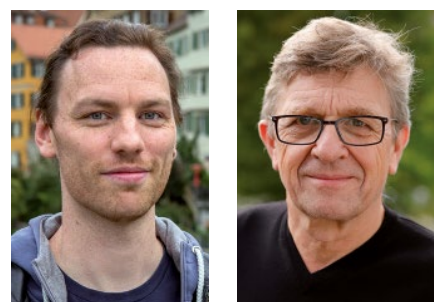

Moritz Koch (links) und Karl Forchhammer

Korrespondenzadresse:

Prof. Dr. Karl Forchhammer

Mikrobiologie/Organismische Interaktion Universität Tübingen

Auf der Morgenstelle 28

D-72076 Tübingen

karl.forchhammer@uni-tuebingen.de 